\title{
Extremely high isolated maternal alkaline phosphatase serum concentration -2 case reports
}

\begin{abstract}
Alkaline Phosphatase is a group of isoenzymes produced by liver, bones, kidneys, small intestine and placenta. It appears in maternal serum between the 15 th and the 26th weeks and increases during the third trimester. Usually, ALP production in maternal serum is not major and total serum ALP level remains normal. Marked elevation of serum ALP may be caused by liver or bone pathology such as malignancy, extra hepatic biliary obstruction, and intrahepatic cholestasis. We report 2 cases of markedly elevated placental isoenzymes of ALP in the third trimester in otherwise uncomplicated pregnancies. In both cases, we have excluded liver, bone, kidney, thyroid, parathyroid, other immunological diseases, and other obstetrics complications. The serum ALP returned to normal level after delivery. We conclude that when markedly raised serum ALP concentration is present during pregnancy, differential diagnosis of other pathology must be excluded. Close monitoring of fetal and maternal conditions, histopathological examination of the placenta, and follow-up of declining ALP concentrations postnatally must be performed. As this condition may have high risk of recurrence in subsequent pregnancies, women should be managed in consultant led clinic.
\end{abstract}

Keywords: alkaline phosphatase, placental isoenzymes, third trimester
Volume 2 Issue 4 - 2017

\author{
Angela Yulia,' Ajith Wijesiriwardana ${ }^{2}$ \\ 'Department of Obstetrics and Gynaecology, North Tyneside \\ General Hopsital, UK \\ ${ }^{2}$ Consultant Obstetrician and Gynaecologist, The Cumberland \\ Infirmary, UK
}

Correspondence: Angela Yulia, Subspecialty Trainee in Maternal and Fetal Medicine, Department of Obstetrics and Gynaecology, University College Hospitals London, Elizabeth Garrett Anderson Wing, 25 Grafton Way, Bloomsbury, London WCIE 6BD, UK, Tel 07738931078, Email ayulia@doctors.org.uk

Received: April 08, 2017 | Published: June 20, 2017

\section{Introduction}

Alkaline phosphatises (ALP) are a group of isoenzymes produced by liver (isoenzymes ALP1-1), bones (isoenzymes ALP2), kidneys, small intestine and placenta (isoenzymes ALP-3). ${ }^{1,2}$ The serum ALP level represents the total amounts of isoenzymes that are released from the tissues into the bloodstream. Placenta ALP is physiologically produced by placenta at the brush border membranes of the syncytiotrophoblast, and its' major function is thought to aid in metabolism and facilitate transport across cell membranes. ${ }^{3}$ It appears in maternal serum between the 15th and the 26th weeks and increases during the third trimester. Usually, ALP production in maternal serum is not major and total serum ALP level remains normal. The mechanism of serum placental ALP increase is not well understood. In postpartum period, ALP isoenzyme activity decreases to the normal level at about 20weeks after delivery. Marked elevation of serum ALP may be caused by liver or bone pathology such as malignancy, extra hepatic biliary obstruction, and intrahepatic cholestasis. ${ }^{2}$ We report 2 cases of markedly elevated placental isoenzymes of ALP in the third trimester in otherwise uncomplicated pregnancies. In both cases, serum ALP returned to normal level after delivery.

\section{Case reports}

Two low-risk primigravida age 29 (patient A) and 20 (patient B) years attended at around 36 weeks pregnant due generally feeling unwell. After general examination and investigations, single significant elevation of serum ALP concentration was detected (5122 and $3017 \mathrm{U} /$ litre respectively).

\section{Case I}

A fit and well 29-year-old primigravida was reviewed at the pregnancy assessment unit due to itchy hands and was suspected to have obstetric cholestasis at 36 weeks and 3 days gestations. Her obstetrics history was unremarkable and gestational age was determined by dating ultrasound scan. After general examination and investigations, significant elevation of serum ALP concentration was detected (5122 U/litre). Other laboratory tests showed that all blood parameters and renal, hepatic, and endocrine functions were normal. No systemic immune disease was detected. Serum ALP electrophoresis showed normal ALP level of liver and bone origin, whereas placental isoenzymes were elevated. She was admitted to the antenatal ward for observation. Ultrasound scan of the fetus revealed normal growth, liquor volume and doppler flow. The fetus was monitored daily with cardiotocography. Abdominal and liver ultrasound scan was unremarkable. A gradual elevation of ALP level was noted on repeated blood tests. On the 38th week of pregnancy, a healthy girl was delivered by Caesarean section due to failed induction of labour. The histopathological examination revealed a $730 \mathrm{~g}$ placenta which showed mild chronic intervillositis of uncertain aetiology. The measurements of the placenta were adequate for the gestational age and neonate body weight. No area of infarction was observed. At the 3rd post-operative day, with a normal course of puerperium, the patient and baby were discharged home. A repeat serum ALP level one week post delivery showed marked decrease in ALP levels. Serum ALP level returned to normal at 6 weeks post-partum.

Other laboratory and radiological tests results were normal. Serum ALP electrophoresis showed normal ALP level of liver and bone origin, whereas placental isoenzymes were elevated. As gradual elevation of ALP levels were noted (Figure 1), she had induction of labour (IOL) at 38weeks. Patient a had Caesarean section due to failed IOL. Healthy infant was delivered. The histopathological examinations of the placenta were unremarkable apart from mild chronic intervillositis of uncertain aetiology in patient A. Serum ALP level returned to normal at 6 weeks post-partum. 


\section{Case 2}

A fit and well 20-year-old primigravida who was 36 weeks pregnant was reviewed in pregnancy assessment unit due to generally feeling unwell. Her clinical history and examination was unremarkable. Blood tests were normal apart from single elevated serum ALP of 3017U/1. Five days previously, her ALP levels was 764 U/1. Other blood tests, such as full blood count, coagulation tests, kidney, liver, and thyroid function tests were all normal. Abdominal and liver ultrasound examination showed no hepatic, biliary tract or renal abnormalities. Obstetrical ultrasonography examination and Doppler blood flow remained normal. The placenta was normal in appearance and size sonographically. She was followed up for one week with daily fetal monitoring using cardiotocography. Serum ALP electrophoresis showed normal ALP level of liver and bone origin, whereas placental isozyme was elevated. Labour was induced at 38 weeks and 1 day gestation using 2 doses of Prostin (3mg 6 hours apart) and she had a normal delivery of healthy female infant. Postpartum examination of the placenta showed a mature placenta, and there was no evidence of infarction site or gross abnormality. Histological examination of placenta was unremarkable. At day 10 postpartum, the alkaline level has fallen to $1010 \mathrm{U} / \mathrm{L}$. Serum ALP level returned to normal at 6 weeks post-partum Figure 1.

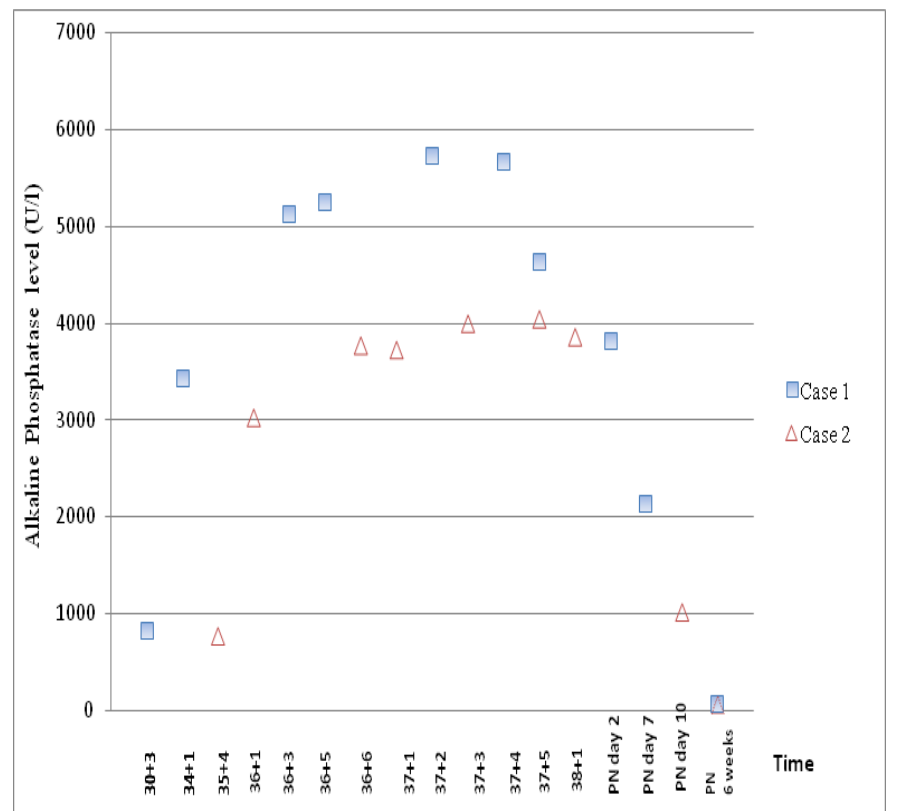

Figure I Alkaline phosphatase levels for both Cases I and 2.

$\mathrm{PN}$ - Days post-natal

\section{Discussion}

The above two cases represents markedly elevated ALP levels, mainly due to placental origin. The mechanism responsible for the markedly elevated serum ALP due to placental origin still remains unknown. ${ }^{2}$ Review of the literature suggests a probable correlation between significantly raised ALP levels and several perinatal complications such as low birth weight, pre-eclampsia and preterm delivery. ${ }^{4,5}$ One study proposed that high level of ALP may cause damage to the chorionic cells as a result of placental insultthe same way in which myocardial enzymes are released into the systemic circulation from a damaged myocardial tissue. ${ }^{3}$ Although this event did not cause any adverse outcome in our cases, it still remain an unresolved question whether a markedly elevated serum ALP of placental origin affect the overall pregnancy outcome, and further studies are needed to prove these association.

A genetic abnormality has been suspected in one case report by. ${ }^{6}$ In this case, the increased in ALP levels in the third trimester of pregnancy was noted in the patient's relatives, suggesting a genetically determined anomaly, which is provoked by pregnancy. The patient herself has pregnancy affected by gestational diabetes mellitus. After delivery, serum ALP levels decreased but did not return to the normal range. This case report concluded that gestational diabetes mellitus did not seem to be associated with an elevated level of serum ALP, but could be the result of genetic anomaly mentioned earlier.

Another case reporting an unusual elevation in ALP levels of placental origin in the last trimester of pregnancy was described. In this case, the only positive finding was that there was infarction in $15 \%$ of the placenta. ${ }^{1}$ However, this insignificant degree of placental infarction is quite common and this did not explain the significant elevation of ALP levels.

With regard to both of our cases, we have excluded liver, bone, kidney, thyroid, parathyroid, and other immunological diseases. Obstetrics complications such as intrauterine growth retardation, preeclampsia, and placenta abruption were also ruled out. There were slow decline in the serum ALP levels following delivery in both cases, and this finding is similar to once case described by. ${ }^{7,8}$ They discussed that a slow decline in the serum ALP level following delivery is a typical characteristic of placental isoenzymes. Among all the ALP isoenzymes, placental isoenzymes activity has the longest half-life of approximately 7days, followed by liver isoenzymes and the shortest half-life is bone isoenzymes, with half-life of 1-2days.

When markedly raised serum ALP concentration is present during pregnancy, differential diagnosis of other pathology must be excluded. Close monitoring of fetal and maternal conditions, histopathological examination of the placenta, and follow-up of declining ALP concentrations postnatally must be performed. ${ }^{2}$ One hypothesis we can derived from this report is that mild chronic infection of the placenta may be responsible for the markedly raised ALP level in pregnancy, however more research is needed to confirm this relationship. As this condition may have high risk of recurrence in subsequent pregnancies, women should be managed in consultant led clinic Figure 1.

\section{Acknowledgements}

None.

\section{Conflict of interest}

Author declares that there is no conflict of interest.

\section{References}

1. Vongthavaravat V, Nurnberger MM, Balodimos $\mathrm{N}$, et al. Isolated elevation of serum alkaline phosphatase level in an uncomplicated pregnancy: a case report. Am J Obstet Gynecol 2000;183(2):505-506.

2. Boronkai A, Than NG, Magenheim R, et al. Extremely high maternal alkaline phosphatise serum concentration with syncytiotrophoblastic origin. J Clinl Pathol. 2005;58(1):72-72.

3. Meyer RE, Thompson SJ, Addy CL, et al. Maternal serum placental alkaline phosphatase level and risk for preterm delivery. Am J Obstet Gynecol. 1995;173(1):181-186. 
4. Goldenberg RL, Iams JD, Mercer BM, et al. The preterm prediction study: toward a multiple marker test for spontaneous preterm birth. Am J Obstet Gynecol. 2001;185(3):643-651.

5. Moawad AH, Goldenberg RL, Mercer B, et al. The preterm prediction study: the value of serum alkaline phosphatise, a-fetoprotein, plasma corticoprotein-releasing hormone, and other serum markers for the prediction of spontaneous preterm birth. Am J Obstet Gynecol. 2002;186(5):990-996.

6. J Wojcicka-Bentyn. Extremely elevated activity of serum alkaline phosphatase in gestational diabetes: a case report. Am J Obstet Gynecol. 2004;190(2):566-567.
7. Bashiri A, Katz O, Maor E, et al. Positive placental staining for alkaline phosphatise corresponding with extreme elevation of serum alkaline phosphatase during pregnancy. Arch Gynecology Obstetrics. 2007;275(3):211-214.

8. Celik H, Tosun M, Cetinkaya MB, et al. Markedly elevated serum alkaline phosphatase level in an uncomplicated pregnancy. J Matern Fetal Neonatal Med. 2009;22(8):705-707. 